\section{Efecto de la electroestimulación percutánea del nervio tibial en pacientes con enuresis monosintomática. Experiencia inicial en México}

Pérez-Martínez C, ${ }^{1,2}$ Palacios-Galicia JL, ${ }^{1,3}$ Vargas-Díaz IB, ${ }^{2}$ Cruz-Gómez Y1,3 $^{1,3}$

\section{Resumen}

OBJETIVO: Analizar el efecto de seis sesiones de neuromodulación por electroestimulación del nervio tibial posterior en pacientes con enuresis monosintomática resistente al tratamiento y describir los factores de riesgo de la técnica.

MATERIALES Y MÉTODOS: Ensayo clínico, aleatorizado, controlado y prospectivo, efectuado en pacientes de 6 a 15 años de edad, con antecedente de enuresis monosintomática de seis meses de evolución, sin reacción al tratamiento de primera y segunda línea. Los pacientes se asignaron al azar a dos grupos: 1) grupo activo (con electroestimulación) y 2) grupo placebo (sin electroestimulación), a quienes se aplicaron seis sesiones de neuromodulación por electroestimulación del nervio tibial posterior (grupo activo) o seis sesiones simuladas (grupo placebo). Se analizaron las variables de edad, nivel socioeconómico, sexo, evolución de la enuresis, diario vesical (antes y después del tratamiento) y uroflujometría. Para determinar la normalidad de los datos se aplicó la prueba de Shapiro-Wilk. La comparación entre grupos por sexo y tipo de tratamiento se realizó con la prueba paramétrica t de Student.

RESULTADOS: Se estudiaron 8 pacientes: 4 niñas y 4 niños, con promedio de edad de $10.6 \pm 1.6$ años. La edad y el sexo no mostraron diferencias estadísticamente significativas. La mejoría de los pacientes (noches secas) fue de $80 \pm 7.5 \%$ en el grupo activo vs $3.7 \pm 1.2 \%$ en el grupo placebo $(p<0.001)$. Los efectos adversos fueron escasos.

CONCLUSIONES: La neuromodulación por electroestimulación del nervio tibial posterior durante seis sesiones es efectiva en el tratamiento de pacientes con enuresis monosintomática resistente al tratamiento; es una técnica de bajo costo, fácilmente reproducible y con efectos adversos casi nulos.

PALABRAS CLAVE: Enuresis; nervio tibial; vejiga.
${ }^{1}$ Doctorado en Ciencias Biológicas, Universidad Autónoma de Tlaxcala, Tlaxcala.

${ }^{2}$ Centro de Urología Avanzada CURA, Delicias, Chihuahua.

${ }^{3}$ Centro Tlaxcala de Biología de la Conducta, Universidad Autónoma de Tlaxcala, Tlaxcala.

Recibido: agosto 2017

Aceptado: noviembre 2017

Correspondencia

Carlos Pérez Martínez

carlosperezm@prodigy.net.mx

Este artículo debe citarse como Pérez-Martínez C, Palacios-Galicia JL, Vargas-Díaz IB, Cruz-Gómez Y. Efecto de la electroestimulación percutánea del nervio tibial en pacientes con enuresis monosintomática. Experiencia inicial en México. Rev Mex Urol. 2018 ene-feb;78(1):19-27.

DOI: https://doi.org/10.24245/revmexurol.v78i1.1719 
Rev Mex Urol. 2018 Jan-Feb;78(1):19-27.

\section{The effect of percutaneous tibial nerve stimulation on patients with monosymptomatic enuresis: An initial experience in Mexico}

Pérez-Martínez C, ${ }^{1,2}$ Palacios-Galicia JL, ${ }^{1,3}$ Vargas-Díaz IB, ${ }^{2}$ Cruz-Gómez $\mathrm{Y}^{1,3}$

Abstract

OBJECTIVE: The aim of our study was to evaluate the effect of six sessions of posterior tibial nerve stimulation on patients with treatmentrefractory monosymptomatic enuresis and to describe the risk factors of the technique.

MATERIALS AND METHODS: A prospective, randomized, controlled clinical trial was conducted on patients between 6 and 15 years of age that presented with monosymptomatic enuresis of six-month progression that did not respond to first-line or second-line treatments. The patients were randomly assigned to one of two groups: 1) active group (with electrical stimulation) and 2) placebo group (without electrical stimulation). Six posterior tibial nerve stimulation sessions were applied to the active group patients and six sham sessions to the placebo group patients. The study variables were age, sex, socioeconomic status, enuresis progression, bladder diary (before and after treatment), and uroflowmetry. Data normality was determined through the ShapiroWilk test and the Student's t test was employed in the comparisons of sex and treatment type between the groups.

RESULTS: Eight patients were recruited for the study, 4 boys and 4 girls, and their mean age was $10.6 \pm 1.6$ years. There was no statistically significant difference in age or sex between the two groups. Patient improvement ("dry" nights) was $80 \pm 7.5 \%$ in the active group vs $3.7 \pm 1.2 \%$ in the placebo group $(p<0.001)$. There were very few adverse effects.

CONCLUSIONS: The effect of six sessions of neuromodulation through posterior tibial nerve stimulation as treatment for refractory monosymptomatic enuresis was effective, low-cost, easily reproducible, and had almost no adverse effects.

KEYWORDS: Enuresis; Tibial nerve; Bladder.

\section{ANTECEDENTES}

La enuresis es la incontinencia urinaria infantil intermitente durante el sueño. ${ }^{1,2}$ El diagnóstico se establece en infantes mayores de 6 años de edad, ${ }^{3}$ con más de un episodio de pérdida de orina mensual. Se clasifica en enuresis infrecuente o significativa cuando ocurren menos o más de 
cuatro episodios por semana, respectivamente. ${ }^{2}$ La prevalencia de enuresis global es de 5 a $15 \% \%^{4,5}$ y en México se ha estimado de 4 a $7 \% .{ }^{6,7}$

La repercusión psicosocial de la enuresis es un tema relevante, los infantes afectados muestran baja autoestima y cambios conductuales secundarios; por tanto, requieren tratamiento multidisciplinario y multimodal..$^{5,8}$ Se ha reportado que solo $55 \%$ de los padres buscan ayuda médica para sus hijos. ${ }^{9}$ Cuando los padres son de bajo nivel educativo, los infantes tienen 4.8 veces más riesgo de no ser atendidos por los médicos. ${ }^{8,9}$ Los niños con enuresis, cuyas familias sufren pobreza y tienen bajo nivel educativo, suelen recibir tratamiento en forma tardía, hasta que la severidad de los síntomas o su cronicidad hacen intolerable la alteración o, bien, porque ellos mismos solicitan atención médica..$^{8,9}$

Según el tiempo de evolución, la enuresis puede ser primaria o secundaria ${ }^{1,2}$ y de acuerdo con las comorbilidades y la reacción al tratamiento (diferencias clínicas que indican diversos orígenes de la enfermedad) ${ }^{2}$ se clasifica en no monosintomática (ENMS) y monosintomática (EMS).

Los pacientes con enuresis no monosintomática manifiestan síntomas de la vía urinaria baja como: disfunción vesical, síndrome de vejiga hiperactiva, disinergia detrusor-esfínter, entre otros. ${ }^{1}$ En contraste, quienes padecen enuresis monosintomática no manifiestan síntomas de la vía urinaria baja ni disfunción vesical; sin embargo, tienen pérdida de orina nocturna, principalmente durante el sueño, ${ }^{1}$ incluso se considera enuresis monosintomática cuando la incontinencia infantil es diurna. ${ }^{1,2}$

El tratamiento de la enuresis monosintomática de primera línea consiste en terapia conductual y dietética (control del consumo de líquidos, hábitos para vaciar la vejiga, etc. $)^{10,11}$ y el de segunda línea implica la adición de psicoterapia, prescripción de medicamentos (desmopresina, imipramina y anticolinérgicos), biorretroalimentación y alarmas, o su combinación. ${ }^{11}$ Hoy día no existe una descripción clara de la tercera línea de tratamiento en pacientes con enuresis y resistencia al mismo; ${ }^{10,11}$ sin embargo, en estos casos puede considerarse la neuromodulación. ${ }^{5}$

Las técnicas más frecuentes de neuromodulación en pacientes con enuresis son la electroestimulación transcutánea parasacral, la neuromodulación por electroestimulación de las raíces sacras (InterStim $\left.{ }^{\circledR}\right)^{12}$ y la neuromodulación por electroestimulación del nervio tibial posterior (PTNS, por sus siglas en inglés). ${ }^{13,14}$

La eficacia de la neuromodulación por electroestimulación del nervio tibial posterior varía de 37.3 a $81.8 \%$ en pacientes con síndrome de vejiga hiperactiva no neurógena; ${ }^{13}$ por tanto, se ha difundido su indicación en adultos y niños con vejiga hiperactiva neurógena y no neurógena. ${ }^{15}$ Hasta la fecha existen pocos estudios prospectivos, controlados, de la neuromodulación por electroestimulación del nervio tibial posterior en pacientes con enuresis monosintomática. ${ }^{16,17}$

Por lo anterior, el objetivo de este estudio es analizar el efecto de seis sesiones de neuromodulación por electroestimulación del nervio tibial posterior en pacientes con enuresis monosintomática resistente al tratamiento y describir los factores de riesgo de la técnica.

\section{MATERIALES Y MÉTODO}

Ensayo clínico, aleatorizado, controlado y prospectivo, efectuado en pacientes de sexo femenino y masculino, de 6 a 15 años de edad, con antecedente de enuresis monosintomática de seis meses de evolución, sin reacción al tratamiento de primera y segunda línea. Los pacientes fueron asignados al azar al grupo activo (estudio) 
o al grupo placebo. Puesto que los pacientes eran niños, los padres o tutores firmaron el consentimiento informado.

Grupo activo: los pacientes recibieron un total de seis sesiones de 30 minutos de neuromodulación por electroestimulación del nervio tibial posterior, tres sesiones por semana. ${ }^{13}$ Se les insertó una aguja de calibre $34 \times 3 "$, de acero inoxidable, en un punto de 3 a $4 \mathrm{~cm}$ cefálico al maléolo interno del pie dominante, inmediatamente posterior al borde de la tibia. ${ }^{18}$ La aguja se conectó a un estimulador neuromuscular EMS+2 (Staodyn, Inc; Longmont Colorado, USA), para administrar una corriente eléctrica cuadrada pulsada de $9 \mathrm{~V}$ y $20 \mathrm{~Hz}$, con una duración del pulso de $200 \mu$ s. La corriente se ajustó de 1 a $9 \mathrm{~mA}$, según el umbral de dolor del paciente o cuando percibió una respuesta fisiológica (flexión plantar del primer ortejo, extensión en abanico de los dedos del pie, fasciculación del bíceps sural y parestesias en el pie). Grupo placebo: los pacientes recibieron un total de 6 sesiones de media hora de una PTNS simulada, 3 sesiones por semana. Se les insertó una aguja de 2 a $3 \mathrm{~mm}$ en el punto tibial $y$ aunque el electroestimulador se mantuvo encendido, no se administró corriente eléctrica. ${ }^{19}$

Se analizaron las variables de edad, sexo, anotaciones en el diario vesical, tiempo de evolución de la enuresis, medicamentos prescritos previamente, uroflujometría para descartar obstrucción infravesical, examen general de orina y urocultivo. Se registraron datos demográficos como: nivel socioeconómico y educativo de los padres o tutores, y maltrato infantil como rubro informativo. El diario vesical y la uroflujometría se realizaron antes del tratamiento. Un mes después se repitió el diario vesical.

Criterios de exclusión: pacientes con enuresis en tratamiento activo y quienes finalizaron el tratamiento farmacológico un mes antes del estudio, infantes con malformaciones congénitas genitourinarias y del sistema nervioso, antecedentes oncológicos y de cirugía pélvica, fracturas pélvicas y de columna vertebral, infección de vías urinarias, trastornos mentales o con diabetes mellitus.

Para el análisis estadístico se recabó la información en una base de datos de Excel y los resultados se procesaron con el programa SPSS 10.1. Para determinar la normalidad de los datos se aplicó la prueba de Shapiro-Wilk. La comparación entre grupos por sexo y tipo de tratamiento se realizó con la prueba paramétrica t de Student.

\section{RESULTADOS}

Se registraron 8 pacientes: 4 niñas y 4 niños, distribuidos equitativamente en cada grupo. La media de edad fue de $10.6 \pm 1.6$ años, sin diferencias estadísticamente significativas entre los grupos y sexo $(\mathrm{p}>0.05)$. El tiempo promedio de evolución de la enuresis fue de $67.5 \pm 19.2$ meses, también sin diferencias significativas entre los grupos ( $p>0.05)$.

Un mes después del tratamiento con neuromodulación por electroestimulación del nervio tibial posterior, los pacientes del grupo activo versus placebo mostraron mejor control de la enuresis ("noches secas"): $80 \pm 7.5$ vs $3.7 \pm$ $1.2 \%$, respectivamente $(\mathrm{p}<0.001)$. No se encontró diferencia significativa en el volumen miccional antes y después del procedimiento en ambos grupos (Cuadro 1). Los efectos adversos más frecuentes fueron: temor a la punción ( $\mathrm{n}=$ 1) y ligera molestia en el sitio de la punción ( $=3$ ), sin observar diferencias significativas entre ambos grupos (Cuadro 2). Todos los pacientes fueron de bajo nivel socioeconómico, con padres o tutores de bajo nivel educativo, además de retraso en el tratamiento y antecedente de maltrato familiar (Cuadro 3). 
Pérez-Martínez C y col. Electroestimulación percutánea del nervio tibial

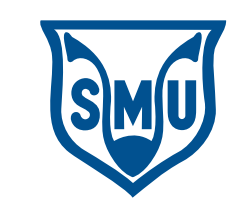

Cuadro 1. Reacción de los pacientes con seis sesiones de PTNS

\begin{tabular}{|c|c|c|c|}
\hline Variable & $\begin{array}{c}\text { Grupo activo } \\
n=4\end{array}$ & $\begin{array}{c}\text { Grupo placebo } \\
n=4\end{array}$ & $\mathbf{p}$ \\
\hline Edad media & $\begin{array}{c}10.5 \text { años } \\
( \pm 1.3)\end{array}$ & $\begin{array}{c}10.7 \text { años } \\
( \pm 1.8)\end{array}$ & 0.780 \\
\hline Tiempo de evolución de la enuresis & $\begin{array}{c}66 \text { meses } \\
( \pm 15.8)\end{array}$ & $\begin{array}{c}69 \text { meses } \\
( \pm 22.6)\end{array}$ & 0.932 \\
\hline Noches secas antes del tratamiento & $\begin{array}{l}12.5 \% \\
( \pm 7.5)\end{array}$ & $\begin{array}{l}6.3 \% \\
( \pm 3.7)\end{array}$ & 0.292 \\
\hline Noches secas 1 mes después del tratamiento & $\begin{array}{l}92.5 \% \\
( \pm 1.4)\end{array}$ & $\begin{array}{c}10 \% \\
( \pm 3.5)\end{array}$ & $<0.001$ \\
\hline Mejoría & $\begin{array}{c}80 \% \\
( \pm 7.3)\end{array}$ & $\begin{array}{l}3.7 \% \\
( \pm 1.2)\end{array}$ & $<0.001$ \\
\hline Volumen miccional antes del tratamiento & $\begin{array}{c}204.3 \mathrm{cc} \\
( \pm 84.8)\end{array}$ & $\begin{array}{l}110.5 \text { cc } \\
( \pm 15.5)\end{array}$ & 0.369 \\
\hline Volumen miccional 1 mes después del tratamiento & $\begin{array}{c}229.2 \text { cc } \\
( \pm 91.3)\end{array}$ & $\begin{array}{c}114 \mathrm{cc} \\
( \pm 15.7)\end{array}$ & 0.314 \\
\hline
\end{tabular}

PTNS: neuromodulación por electroestimulación percutánea del nervio tibial posterior. Los datos representan las medias \pm error estándar; prueba t de Student (IC95\%).

Cuadro 2. Efectos adversos durante las sesiones de PTNS $(\mathrm{n}=8)$

\begin{tabular}{|c|c|c|c|c|c|c|}
\hline Caso & Sexo & Grupo & $\begin{array}{l}\text { Temor a la } \\
\text { punción }\end{array}$ & $\begin{array}{l}\text { Dolor en el sitio } \\
\text { de punción }\end{array}$ & $\begin{array}{c}\text { Sangrado en el sitio de } \\
\text { punción }\end{array}$ & $\begin{array}{l}\text { Dolor o molestia } \\
\text { durante la PTNS }\end{array}$ \\
\hline 1 & 2 & Activo & 1 & 2 & 1 & 2 \\
\hline 2 & 2 & Activo & 4 & 2 & 1 & 1 \\
\hline 3 & 1 & Activo & 2 & 1 & 2 & 1 \\
\hline 4 & 1 & Activo & 1 & 1 & 1 & 1 \\
\hline Media & & & 2 & 1.5 & 1.25 & 1.25 \\
\hline 5 & 1 & Placebo & 2 & 1 & 1 & 1 \\
\hline 6 & 1 & Placebo & 1 & 2 & 1 & 1 \\
\hline 7 & 2 & Placebo & 2 & 1 & 1 & 1 \\
\hline 8 & 2 & Placebo & 1 & 1 & 1 & 2 \\
\hline Media & & & 1.5 & 1.25 & 1 & 1.25 \\
\hline Significación & & & $p=0.595$ & $p=0.374$ & $p=0.374$ & $p=1.000$ \\
\hline
\end{tabular}

PTNS: neuromodulación por electroestimulación percutánea del nervio tibial posterior; sexo femenino = 2; sexo masculino $=1$. Escala análoga: nulo $=1$, leve $=2$, moderado $=3$, severo $=4$. Significación estadística $=p$ grupo activo $v s$ placebo, para prueba t de Student (IC95\%).)

\section{DISCUSIÓN}

La enuresis monosintomática es una alteración que afecta la calidad de vida de los niños y adultos. Se ha demostrado que el tratamiento conductual y farmacológico es poco efectivo, pues la mayoría de los pacientes tiene recidiva.

La neuromodulación en el control de los síntomas de la enuresis monosintomática es una 
Cuadro 3. Nivel socioeconómico de los pacientes

\begin{tabular}{|c|c|c|c|c|c|c|c|}
\hline Caso & $\begin{array}{l}\text { Edad } \\
\text { (años) }\end{array}$ & Sexo & Grupo & $\begin{array}{l}\text { Evolución de la } \\
\text { enuresis (meses) }\end{array}$ & $\begin{array}{l}\text { Escolaridad del } \\
\text { padre o tutor }\end{array}$ & $\begin{array}{l}\text { Antecedente de } \\
\text { maltrato infantil }\end{array}$ & $\begin{array}{c}\text { Rendimiento } \\
\text { escolar }\end{array}$ \\
\hline 1 & 14 & 2 & Activo & 108 & Primaria & Sí & Bajo \\
\hline 2 & 9 & 2 & Activo & 48 & Secundaria & Sí & Regular \\
\hline 3 & 8 & 1 & Activo & 36 & Secundaria & No & Bajo \\
\hline 4 & 11 & 1 & Activo & 72 & Primaria & Sí & Alto \\
\hline 5 & 6 & 1 & Placebo & 12 & Licenciatura & Sí & Bajo \\
\hline 6 & 15 & 1 & Placebo & 120 & Primaria & No & Regular \\
\hline 7 & 10 & 2 & Placebo & 60 & Secundaria & Sí & Bajo \\
\hline 8 & 12 & 2 & Placebo & 84 & Primaria & Sí & Bajo \\
\hline
\end{tabular}

Sexo femenino $=2 ;$ sexo masculino $=1$.

técnica efectiva, principalmente la modalidad por electroestimulación percutánea del nervio tibial posterior, que es superior a la electroestimulación transcutánea parasacral (8.5 a $68.5 \%),{ }^{20-22}$ aunque esta última tiene la ventaja de no ser invasiva..$^{8,21,23-25}$ Hasta la fecha solo existe un ensayo controlado de enuresis monosintomática, ${ }^{31}$ lo que sugiere la escasa investigación de la neuromodulación por electroestimulación percutánea del nervio tibial posterior en pacientes con enuresis monosintomática resistente al tratamiento.

La mayor parte de los estudios consiste en revisiones y guías de tratamiento enfocados a la enuresis no monosintomática (NMS), especialmente para el control de pacientes con vejiga hiperactiva. La International Children's Continence Society ${ }^{10}$ tampoco señala a la neuromodulación para el tratamiento de la enuresis resistente al tratamiento y se limita al uso de alarmas y prescripción de fármacos anticolinérgicos y de hormona antidiurética, ${ }^{10}$ que provocan efectos secundarios.

Nuestro protocolo de seis sesiones de electroestimulación percutánea del nervio tibial posterior (PTNS) en pacientes con enuresis monosintomáticos resistente al tratamiento mostró eficacia en $80 \%$ de los casos. Este resultado es similar al del estudio de Neveus y su equipo de trabajo, quienes aplicaron 12 sesiones de electroestimulación del nervio tibial posterior $(78.6 \%)^{14}$ y es mayor que las cifras obtenidas con otras técnicas de neuromodulación, como la neuroestimulación percutánea sacra (InterStim $\left.{ }^{\circledast}\right)$, cuya eficacia es de $60 \%{ }^{12,26,27}$ Esta última representa una técnica costosa, además de requerir procedimientos quirúrgicos, por lo que es poco disponible para los pacientes y el personal médico, incluso ha sido desplazada por técnicas menos invasivas. ${ }^{28}$

Aunque la neuromodulación por electroestimulación percutánea del nervio tibial posterior es una técnica fácilmente reproducible, de bajo costo y tolerable para los niños (pues provoca efectos adversos leves), ha sido subutilizada y poco estudiada. En este estudio, los efectos adversos más frecuentes fueron el temor a la punción y leve incomodidad en el sitio de la punción; sin embargo, ningún paciente abandonó el tratamiento, similar a lo reportado por Gennaro y sus colaboradores, ${ }^{29}$ quienes reportan elevada tolerancia al dolor en los niños que recibieron electroestimulación del nervio tibial posterior. Los estudios de Raheem y su grupo ${ }^{14}$ y de Capitanuci y sus coautores ${ }^{30}$ no reportaron efectos adversos ni abandono del tratamiento, tampoco especifican el método ni el cuestionario aplicado. 
Hasta la fecha se desconocen los mecanismos fisiológicos asociados con la mayor efectividad de la neuromodulación por electroestimulación percutánea del nervio tibial posterior en niños que en adultos. De hecho, cuando McGuire ${ }^{18}$ describió el tratamiento de la electroestimulación del nervio tibial posterior en pacientes con detrusor hiperactivo, los estudios implicados con los mecanismos de acción indicaron que el nervio tibial posterior es un nervio somático mixto sensitivo y motor, además de ser la rama principal del nervio ciático que se origina en los segmentos medulares L5 a S3. En esa zona espinal, la información del nervio tibial converge con la inervación parasimpática de la vejiga y del nervio pudendo; ${ }^{31}$ entonces, el impulso antidrómico aplicado a las raíces anteriores medulares inhibe el centro parasimpático sacro y bloquea el impulso aferente de la hiperactividad del detrusor. ${ }^{32}$ Posteriormente, existe neuroplasticidad en el encéfalo por la aplicación de electroestimulación del nervio tibial posterior en pacientes con hiperactividad del detrusor, cuyo efecto permanece 24 horas después de aplicar el estímulo y de esta manera se explica el mecanismo, mediante potenciales somatosensoriales evocados..$^{33}$ También se han descrito diferentes efectos neuroquímicos medulares y supraespinales provocados por el tratamiento con neuromodulación por electroestimulación del nervio tibial posterior en adultos y niños. ${ }^{34}$ La neuromodulación afecta diferentes áreas supraespinales, principalmente las del aprendizaje. ${ }^{35}$ Recientemente se observó que las redes sensoriales-motoras implicadas en el control de la vejiga urinaria y la locomoción se encuentran altamente integradas, conductual y neurofisiológicamente, y que ambas funciones están moduladas por la aferencia sensorial desde los nervios tibial y pudendo, ${ }^{36}$ lo que explica el proceso de neuromodulación por electroestimulación de ambos nervios. De acuerdo con estos hallazgos, se sugiere que la modulación de la función vesical por electroestimulación del nervio tibial posterior funciona a nivel medular y supraespinal, estimulando interneuronas inhibidoras, específicamente receptores de glutamato y opioides. ${ }^{37}$
Aún se discute si la efectividad mayor de la electroestimulación del nervio tibial posterior en niños se debe a la estimulación del sistema nervioso en desarrollo, cuando algunos circuitos continúan madurando, o porque el sistema nervioso de los niños tiene mayor capacidad de aprendizaje y, por lo tanto, de plasticidad.

En este estudio no encontramos cambios estadísticamente significativos en la capacidad vesical de ambos grupos (activo versus placebo), lo que coincide con lo reportado en otros ensayos. ${ }^{38}$ Esto sugiere que la electroestimulación del nervio tibial posterior no afecta el circuito neuromuscular del vaciamiento vesical. Se requieren estudios adicionales para conocer si la electroestimulación del nervio tibial posterior afecta la manifestación de los síntomas de pacientes con vejiga hiperactiva o hipoactiva, pues se ha observado que la electroestimulación nerviosa en la vejiga es altamente dependiente de la frecuencia, incluso se ha descrito que la frecuencia inhibitoria varía de 3 a 15 Hertz, mientras que la frecuencia de 20 Hertz, o mayor, produce un efecto de excitación en el detrusor. ${ }^{39}$

Se requieren estudios preclínicos y clínicos para esclarecer el efecto fisiológico de la neuromodulación por electroestimulación percutánea del nervio tibial posterior. El efecto de la electroestimulación del nervio tibial posterior perdura a corto y mediano plazo. Así lo demostró un estudio no controlado en niños con disfunciones urinarias, con seguimiento de 2 años. ${ }^{30}$ La demografía de los casos de nuestro estudio mostró maltrato infantil en 6 de los 8 niños, tema que será abordado en una próxima publicación.

\section{CONCLUSIONES}

El tratamiento de neuromodulación por electroestimulación percutánea del nervio tibial en pacientes con enuresis monosintomática resistente al tratamiento muestra mejores resultados 
que otras técnicas, incluida la electroestimulación transcutánea, además de ser fácilmente reproducible y no provocar efectos adversos (casi nulos). Los resultados de nuestro estudio indican que la reacción con seis sesiones de electroestimulación percutánea del nervio tibial es similar a la obtenida con 12 sesiones.

\section{Agradecimientos}

Por su colaboración y trato humano: a la técnica Cinthia Edith López Evens, quien adiestró a los pacientes para completar el cuestionario y el diario vesical, además de ofrecer el tratamiento de neuromodulación por electroestimulación percutánea del nervio tibial.

\section{Fuente de financiamiento}

Los estudios realizados a los voluntarios, el procedimiento de neuromodulación y los materiales implementados fueron donados por Litomovil del Norte S.A. de C.V., sin ninguna limitante, condición o restricción para pacientes o investigadores.

\section{Conflicto de interés}

Los autores de este estudio declaran no tener conflicto de interés.

\section{REFERENCIAS}

1. Nevéus T, Gontard A Von, Hoebeke P, et al. The standardization of terminology of lower urinary tract function in children and adolescents: report from the Standardisation Committee of the International Children's Continence Society. J Urol. 2006;176(1):314-324.

2. Austin PF, Bauer SB, Bower W, et al. The standardization of terminology of lower urinary tract function in children and adolescents: update report from the standardization committee of the International Children's Continence Society. Neurourol Urodyn. 2016;35:471-481.

3. American Psychiatric Association. Manual Diagnóstico $Y$ Estadistico de Transtornos Mentales, DSM-V. Actualización. 5a ed. Arlington, VA: American Psychiatric Association Publishing; 2016. [En línea]. Dirección URL: <http://www. dsm5.org>.
4. van Engelenburg-van Lonkhuyzen $M L$, Bols EM, Benninga MA, Verwijs WA, de Bie RA. Bladder and bowel dysfunctions in 1748 children referred to pelvic physiotherapy : clinical characteristics and locomotor problems in primary, secondary, and tertiary healthcare settings. Eur J Pediatr. 2017;176:207-216.

5. Maternik M, Krzeminska K. The management of childhood urinary incontinence. Pedriatric Nephol. 2015;30:41-50.

6. Caraveo AJJ. Cuestionario breve de tamizaje y diagnóstico de problemas de salud mental en niños y adolescentes: algoritmos para síndromes y su prevalencia en la Ciudad de México. Segunda parte. Salud Mental 2007;30(1):48-55.

7. Feria $\mathrm{M}$, Cárdenas $\mathrm{M}$, Vázquez J, Palacios $\mathrm{L}$, de la Peña $\mathrm{F}$. Guía clínica para el manejo de los trastornos de eliminación (enuresis y encopresis ). In: Berenson S, Del Bosque J, Alfaro J, Medina-Mora M, editores. Guías clínicas para la atención de trastornos mentales. México: Instituto Nacional de Psiquiatría, 2010;pp:5-21.

8. Jesus LE De, Tome A, Cobe D, Camelier P. Psychosocial and respiratory disease related to severe bladder dysfunction and non-monosymptomatic enuresis. J Pediatr Urol. 2016;12:1-6.

9. Schlomer B, Rodriguez E, Weiss D, Copp H. Parental beliefs about nocturnal enuresis causes, treatments, and the need to seek professional medical care. J Pediatr Urol. 2013;9(6):1043-1048.

10. Dodds PR. Re: Evaluation of and Treatment for monosymptomatic enuresis: a standardization document from the International Children's Continence Society: Neveus T, Eggert P, Evans J, et al. J Urol. 2010;184(2):441-447. J Urol 2010;184(2):806-7. doi:10.1016/j.juro.2010.04.0063.

11. Nevéus T. Nocturnal enuresis-theoretic background and practical guidelines. Pediatr Nephol. 2011;26:1207-1214. doi:10.1007/s00467-011-1762-8.

12. Roth TJ, Vandersteen DR, Hollatz P, Inman BA, Reinberg YE. Sacral Neuromodulation for the dysfunctional elimination syndrome: a single center experience with 20 children. J Urol 2008;180(1):306-311. doi:10.1016/j.juro.2008.03.033.

13. Wibisono E, Rahardjo HE. Effectiveness of short term percutaneous tibial nerve stimulation for non-neurogenic overactive bladder syndrome in adults: A meta-analysis. Acta Med Indones. 2015;32:188-200.

14. Raheem AA, Farahat $\mathrm{Y}$, El-gamal O, et al. Role of posterior tibial nerve stimulation in the treatment of refractory monosymptomatic nocturnal enuresis: a pilot study. J Urol. 2013;189(4):1514-1518. doi:10.1016/j.juro.2012.10.059.

15. De Wall LL, Heesakkers J. Effectiveness of percutaneous tibial nerve stimulation in the treatment of overactive bladder syndrome. Res Reports Urol. 2017;9:145-157.

16. Sanford MT, Suskind AM. Neuromodulation in neurogenic bladder. Translacional Androl Urol. 2016;5(1):117-126. doi:10.3978/j.issn.2223-4683.2015.12.01.

17. Boudaoud N, Line A, Chaouadi D, Jolly C, Fiquet CF, Ripert T. Management of refractory overactive bladder in children by transcutaneous posterior tibial nerve stimu- 
Pérez-Martínez C y col. Electroestimulación percutánea del nervio tibial

lation : A controlled study. J Pediatr Urol. 2015;11:1-10. doi:10.1016/j.jpurol.2014.09.013.

18. van Balken MR, Vergunst $H$, Bemelmans BLH. The use of electrical devices for the treatment of bladder dysfunction: a review of methods. J Urol. 2004;172(3):846-851. doi:10.1097/01.ju.0000134418.21959.98.

19. Peters K, Carrico D, Burks F. Validation of a sham for percutaneous tibial nerve stimulation (PTNS). Neurourol Urodyn. 2009;28(1):58-61. doi:10.1002/nau.20585.

20. Jørgensen CS, Kamperis K, Borch L, Borg B, Rittig S. Transcutaneous Electrical Nerve Stimulation in Children with Monosymptomatic Nocturnal Enuresis: A Randomized, Double-Blind, Placebo Controlled Study. J Urol. 2017;198(3):687-693. doi:10.1016/j.juro.2017.04.082.

21. Kajbafzadeh A, Sharifi-Rad L, Mozafarpour S, Ladi-Seyedian S. Efficacy of transcutaneous interferential electrical stimulation in treatment of children with primary nocturnal enuresis: a randomized clinical trial. J Pediatic Nephol. 2015;30(7):1139-1145. doi:10.1007/s00467-014-3039-5.

22. Barroso U, Viterbo W, Bittencourt J, Farias T, Lordêlo P. Posterior tibial nerve stimulation vs parasacral transcutaneous neuromodulation for overactive bladder in children. J Urol. 2013;190(2):673-677. doi:10.1016/j.juro.2013.02.034.

23. Ferroni MC, Chaudhry R, Shen B, Chermansky CJ, et al. Transcutaneous electrical nerve stimulation of the foot: results of a novel at-home, noninvasive treatment for nocturnal enuresis in children. Urology. 2017;101:80-84. doi:10.1016/j.urology.2016.10.023.

24. Patidar N, Mittal V, Kumar M, Sureka SK, Arora S, Ansari MS. Transcutaneous posterior tibial nerve stimulation in pediatric overactive bladder: A preliminary report. J Pediatr Urol. 2015;11(6):351.e1-351.e6. doi:10.1016/j.jpurol.2015.04.040.

25. Lecompte J, Hery G, Guys J, Louis-borrione C. Evaluation of transcutaneous electrical posterior tibial nerve stimulation for the treatment of fecal and urinary leaks in children: Preliminary results. J Pediatr Surg. 2015;50(4):630-633. doi:10.1016/j.jpedsurg.2014.05.033.

26. Dwyer ME, Vandersteen DR, Hollatz P, Reinberg YE. Sacral neuromodulation for the dysfunctional elimination syndrome: a 10-year single-center experience with 105 consecutive children. J Pediatr Urol. 2014;84(4):911-917. doi:10.1016/j.urology.2014.03.059.

27. Stephany HA, Juliano TM, Clayton DB, et al. Prospective evaluation of sacral nerve modulation in children with validated questionnaires. J Urol. 2013;190(4):1516-1522. doi:10.1016/j.juro.2013.01.099.

28. Le N, Kim J. Expanding the role of neuromodulation for overactive bladder: new indications and alternatives to delivery. 2011:25-30. doi:10.1007/s11884-010-0074-3.
29. De Gennaro M, Capitanucci ML, Mosiello G, Zaccara A. Current state of nerve stimulation technique for lower urinary tract dysfunction in children. J Urol. 2011;185(5):15711577. doi:10.1016/j.juro.2010.12.067.

30. Capitanucci ML, Camanni D, Demelas F, Mosiello G, Zaccara A, De Gennaro M. Long-term efficacy of percutaneous tibial nerve stimulation for different types of lower urinary tract dysfunction in children. J Urol. 2009;182(4):2056-2061. doi:10.1016/j.juro.2009.03.007.

31. Yoo PB, Horvath EE, Amundsen CL, Webster GD, Grill WM. Intraurethral activation of excitatory bladder reflexes in persons with spinal cord injury. Conf Proc IEEE Eng Med Biol Soc. 2009;2009:6781-6784. doi:10.1109/ IEMBS.2009.5333982.

32. De Groat WC, Ryall RW. Recurrent inhibition in sacral parasympathetic pathways to the bladder. J Physiol. 1968;196(3):579-591. doi:10.1113/jphysiol.1968. sp008524.

33. Finazzi-Agro E, Rocchi C, Pachatz C, Marfia GA. Percutaneous tibial nerve stimulation produces effects on brain activity: study on the modifications of the long latency somatosensory evoked potentials. Neurourol Urodyn. 2009;28:320-324. doi:10.1002/nau.

34. Boor R, Li L, Goebel B, Reitter B. Subcortical somatosensory evoked potentials after posterior tibial nerve stimulation in children. Brain Dev. 2008;30:493-498. doi:10.1016/j. braindev.2007.06.010.

35. Amend B, Matzel KE, Abrams P, Groat WC De, Sievert K. How does neuromodulation work. Neurourol Urodyn. 2011;30:762-765. doi:10.1002/nau.21096

36. Gad PN, Roy RR, Zhong H, Gerasimenko YP, Taccola G, Edgerton VR. Neuromodulation of the neural circuits controlling the lower urinary tract. Exp Neurol. 2016;285:182189. doi:10.1016/j.expneurol.2016.06.034.

37. Matsuta Y, Mally AD, Zhang F, et al. Contribution of opioid and metabotropic glutamate receptor mechanisms to inhibition of bladder overactivity by tibial nerve stimulation. Am J Physiol Regul Integr Comp Physiol. 2013;305:126-133. doi:10.1152/ajpregu.00572.2012.

38. Kabay SC, Kabay S, Yucel M, Ozden H. Acute urodynamic effects of percutaneous posterior tibial nerve stimulation on neurogenic detrusor overactivity in patients with Parkinson's disease. Neurourol Urodyn. 2009;28:62-67. doi:10.1002/nau.20593

39. Mcgee MJ, Grill WM. Modeling the spinal pudendo-vesical reflex for bladder control by pudendal afferent stimulation. J Comput Neurosci. 2016;40(3):283-296. doi:10.1007/ s10827-016-0597-5.Modeling. 